\title{
A novel chemical, STF-083010, reverses tamoxifen-related drug resistance in breast cancer by inhibiting IRE1/XBP1
}

\author{
Jie Ming ${ }^{1, *}$, Shengnan Ruan ${ }^{1, *}$, Mengyi Wang ${ }^{1}$, Dan Ye ${ }^{1}$, Ningning Fan ${ }^{2}$, Qingyu Meng ${ }^{3}$, \\ Bo Tian ${ }^{4,5}$, Tao Huang ${ }^{1}$ \\ ${ }^{1}$ Department of Breast and Thyroid Surgery, Union Hospital, Tongji Medical College, Huazhong University of Science and \\ Technology, Wuhan 430022, P.R. China \\ ${ }^{2}$ Department of Breast and Thyroid Surgery, Luoyang Central Hospital, Zhengzhou University, Luoyang, Henan 471009, \\ P.R. China \\ ${ }^{3}$ Department of Radiation Oncology, Peking Union Medical College hospital, Beijing 100730, P.R. China \\ ${ }^{4}$ Department of Neurobiology, Tongji Medical College, Huazhong University of Science and Technology, Wuhan 430030, \\ P.R. China \\ ${ }^{5}$ Key Laboratory of Neurological Diseases of Hubei Province, Tongji Medical College, Huazhong University of Science and \\ Technology, Wuhan 430030, P.R. China \\ *These authors have contributed equally to this work
}

Correspondence to:

Jie Ming, e-mail: mingjiewh@126.com

Tao Huang, e-mail: huangtaowh@163.com

Keywords: STF-080310, tamoxifen, breast cancer, IRE I/XBPI

Received: May 21, $2015 \quad$ Accepted: September 23, $2015 \quad$ Published: October 19, 2015

\section{ABSTRACT}

Recent studies show that the unfolded protein response (UPR) within the endoplasmic reticulum is correlated with breast cancer drug resistance. In particular, human X-box binding protein-1(XBP1), a transcription factor which participates in UPR stress signaling, is reported to correlate with poor clinical responsiveness to tamoxifen. In this study, we develop a tamoxifen-resistant MCF-7 cell line by treating the cell line with low concentration of tamoxifen, and we find that XBP1 is indeed up-regulated at both the mRNA and protein levels compared to normal MCF7 cells. STF-083010, a novel inhibitor which specifically blocks the XBP1 splicing, reestablishes tamoxifen sensitivity to resistant MCF-7 cells. Moreover, co-treatment with STF-083010 and tamoxifen can significantly delay breast cancer progression in a xenograft mammary tumor model. We next investigate the expression of XBP1s in over 170 breast cancer patients' samples and the results demonstrate that XBP1s expression level is highly correlated with overall survival in the $\mathrm{ER}^{+}$subgroup, but not in the ER- subgroup, suggesting a potential therapeutic application of XBP1 inhibitors in $\mathbf{E R}^{+}$breast cancer treatment.

\section{INTRODUCTION}

Tamoxifen (TAM) is one of the most frequently used, and effective, endocrine treatment drugs, which can reduce mortality and breast cancer recurrence in patients with hormone receptor positive breast tumors. Unfortunately, up to $50 \%$ of estrogen or progesterone receptor positive breast cancers do not respond to endocrine therapies, displaying de novo or intrinsic resistance. Therefore, the development of novel and efficient therapies for tamoxifen-resistant breast cancer remains a major challenge for breast cancer researchers and clinicians. [1] The unfolded protein response (UPR), a collective set of signaling pathways which is activated by endoplasmic reticulum (ER) stress, has been demonstrated to be one of the most important endocrine treatment-resistant mechanisms and represents a potential therapeutic target for tamoxifen-resistant breast cancer. [2] However, the detailed mechanisms of how the three UPR downstream branches (IRE1, PERK, and ATF6) integrate their cyto-protective and proapoptotic outputs under ER stress, such as hypoxia, starving or tamoxifen treatment, are still unknown. [3] Some studies have suggested that the ER transmembrane kinase/endoribonuclease (RNase) 
IRE1alpha is a key component of the cell-fate switch for UPR-triggered apoptosis or survival $[4,5]$.

XBP1 is reported to be an important regulator of the UPR [5]. It has been shown that only the spliced form of XBP1 (XBP1s) can induce the UPR efficiently. Moreover, $\mathrm{XBP} 1 \mathrm{~s}$ is more stable, easier to transport and a stronger transcriptional factor compared to its unspliced form, XBP1u. [6]

Previous studies have demonstrated that XBP1 expression is increased in estrogen therapy resistant breast cancer cell lines and is co-expressed with the estrogen receptor alpha (ERalpha) in breast tumors. $[7,8]$ Overexpression of XBP1s in ER-positive breast cancer cells leads to estrogen-independent cell growth and reduced sensitivity to growth inhibition induced by tamoxifen and Faslodex independent of a functional p53. [9] The ratios of XBP1s/XBP1u mRNA (indicating enhanced splicing by IRE1) in 100 primary breast cancer patients who received tamoxifen treatment were measured by quantitative RT-PCR, and the result suggests that higher ratios of XBP1s/XBP1u are correlated with poorer survival [10]. Gene expression profile analysis reveals that $\mathrm{XBP} 1 \mathrm{~s}$ acts through transcriptional regulation of the estrogen receptor, the antiapoptotic gene BCL2, and several other genes associated with cell cycle and apoptosis. [11, 12]

XBP1s expression level may be one of the key players in tamoxifen-resistant breast cancer, while IRE1, the upstream nuclease which mediates the splicing of XBP1 pre-mRNA, may be a potential target for reducing resistance. Classical IRE1-XBP1 inhibitors, such as sunitinib and AYP29, not only inhibit the kinase function of IRE1but also activates its endonuclease activity, which has no effect on XBP1 in terms of expression. [13] Intriguingly, a new type of IRE1-XBP1 inhibitor, STF083010, inhibits only IRE1 RNase activity but does not alter the phosphorylation process, thus decreasing XBP1s protein level. [14]

In this study, we demonstrate increased levels of XBP1s both at the mRNA and protein level in a tamoxifen-resistant MCF-7 cell line (termed MCF7TAMR hereafter) compared to normal MCF-7 cells (termed control cells hereafter). Moreover, we also find that STF-083010, a novel inhibitor which specifically blocks XBP1 splicing, can re-establish MCF7-TAMR cells' sensitivity to tamoxifen treatment in vitro. Furthermore, we demonstrate the synergistic effect of STF-083010 and tamoxifen in controlling breast cancer progression in a xenograft murine mammary cancer model. Finally, we investigate XBP1s expression in over 170 breast cancer patients' samples, and show that XBP1s expression is highly correlated with overall survival in $\mathrm{ER}^{+}$breast cancer patients, strongly suggesting a potential therapeutic application of XBP1 inhibitors in breast cancer treatment.

\section{RESULTS}

\section{Establishment of MCF7-TAMR and T47D- TAMR cell lines}

To develop a MCF7-TAMR cell line, we exposed normal MCF-7 cells to a low concentration of tamoxifen $(1 \mu \mathrm{M})$ for 30 days continuously and then compared the viability of these treated cells to control cells at $1 \mu \mathrm{M}$, $2 \mu \mathrm{M}$ and $4 \mu \mathrm{M}$ tamoxifen. The results showed that the MCF7-TAMR cells exhibited significantly less sensitivity to tamoxifen treatment at all three concentrations compare to the control cells (Figure 1a). Microscopic analysis was used to assess the morphological changes upon tamoxifen treatment; we found that control MCF-7 cells displayed a highly round morphology, while the MCF7-TAMR cells showed more branches and displayed a long, flat morphology which were similar to normal tissue epithelia (Figure 1b). We also developed T47D-TAMR cells by exposing control T47D cells to $10 \mu \mathrm{M}$ tamoxifen for 30 days continuously.

\section{Elevated XBP1s level in the MCF7-TAMR and T47D-TAMR cell lines}

To compare XBP1 expression level between control cells and tamoxifen resistant cells, we performed RTPCR to analyze the mRNA level of XBP1s and XBP1u, and the XBP1s/ XBP1u ratio was used as a measure of XBP1 splicing activity. We found that both in MCF7TAMR and T47D-TAMR cells, the XBP1s/ XBP1u ratio was significantly higher than control cells (Figure 1c, Supplementary Figure S1b), suggesting more efficient XBP1 processing. Immunoblot analysis also showed that the XBP1s protein level was significantly higher in MCF7-TAMRcells and T47D-TAMR cells and in line with this, the XBP1 upstream regulator, IRE1, showed higher activity (increased phosphorylation) in MCF7-TAMR cells and T47D-TAMR cells (Figure 1d, Supplementary Figure $\mathrm{S} 1 \mathrm{c})$. We also investigated AKT and Caspase 3 activities, but no significant difference was found between control cells and tamoxifen resistant cells. Interestingly, when we treated MCF7-TAMR cells with $4 \mu \mathrm{M}$ tamoxifen and monitored the spliced XBP1s mRNA level, we found that the XBP1s mRNA was increased in a time dependent manner only in the MCF7-TAMRcells (Figure 1e), suggesting that XBP1's splicing was correlated with the tamoxifen treatment in a time dependent manner.

\section{The IRE1/XBP1 inhibitor, STF-080310, restores tamoxifen sensitivity in MCF7-TAMR cells}

STF-080310 is a novel IRE1/XBP1 inhibitor which can specifically inhibit the RNase activity of IRE1without affecting its kinase function (Figure 2a), and therefore 
a

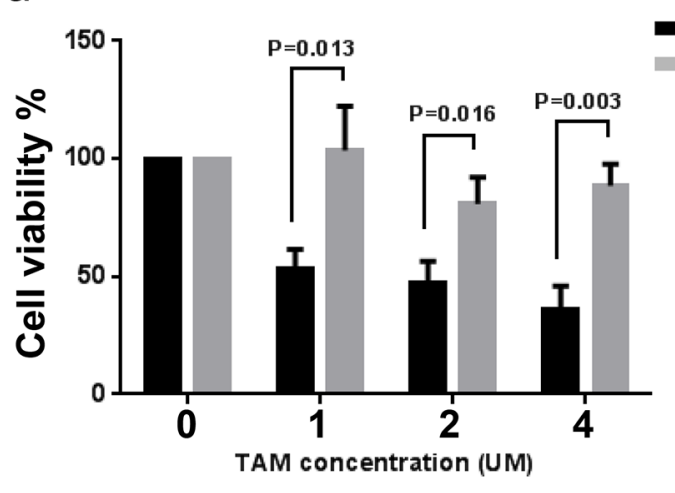

b

C

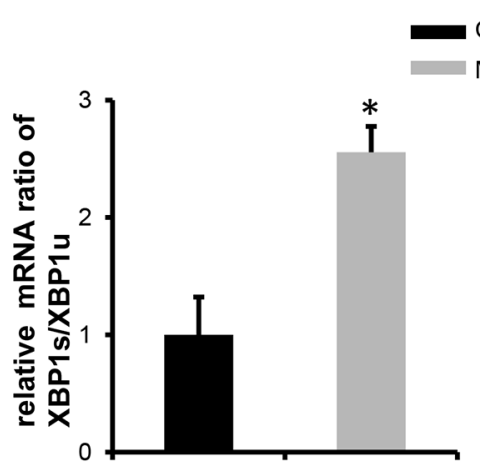

d

XBP1s

XBP1

p-IRE1

IRE1
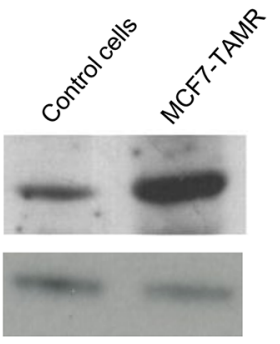

Control cells MCF7-TAMR

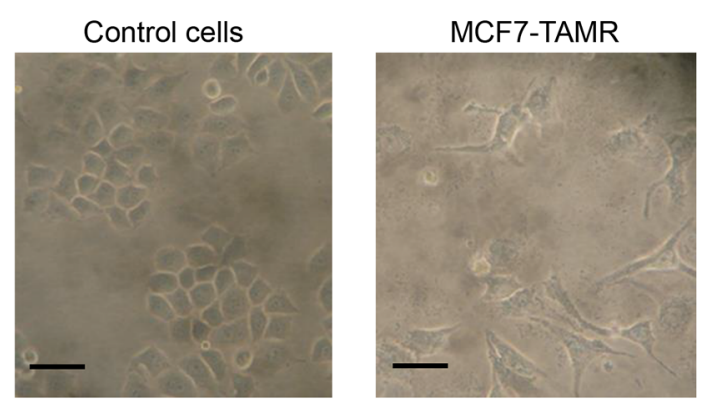

e

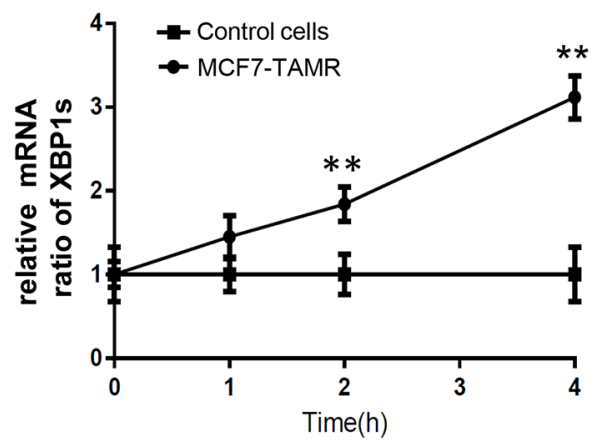

Figure 1: Elevated XBP1s expression level is associated with acquired tamoxifen resistance. a. Long-term treatment (30days) with low tamoxifen concentration induced tamoxifen-resistance in MCF-7 cells, which showed significantly less sensitivity to tamoxifen under different concentrations. Cell viabilities were determined 48 hours after tamoxifen treatment by MTT assay at least 3 times at different conditions, mean survival ( \pm s.e.m.) of control MCF-7 cells and MCF7-TAMR cells are shown in the bar graph. (unpaired, two-sided $t$-test). b. Morphological analysis of control and MCF7-TAMR cells by microscopy. Note the control cells exhibit round shape character, while resistant cells show more branches and flat morphology, which are more similar to normal breast epithelia. Scale bars, $100 \mu \mathrm{m}$. c. mRNA level of XBP1s/XBP1u ratio are determined by RT-PCR in both control and MCF7-TAMR cells. The ratio in MCF7TAMR cells is significantly higher compared to control cells, suggesting a highly active splicing process in MCF7-TAMR cells. ${ }^{*} P<0.05$. (unpaired, two-sided $t$-test). d. Immunoblot analysis of XBP1s and p-IRE1 protein levels in control and MCF7-TAMR cells. Representative results are shown. Note the XBP1s and phosphorylated IRE1expression level in MCF7-TAMR cells are significantly higher than control cells, indicating a more dynamic IRE1-XBP1 axis in MCF7-TAMR cells. The XBP1u, IRE1 and GAPDH are used as loading controls. e. Time dependent analysis of XBP1s mRNA level in MCF7-TAMR cells by RT-PCR. Results from three independent experiments are shown in mean ratio ( \pm s.e.m). Note that the control cells mRNA level are always normalized to 1 in all conditions. ${ }^{*} P<0.05 ; * * P<0.01$. (unpaired, two-sided $t$-test).

can block XBP1 splicing and decrease XBP1s levels. [14]. Thapsigargin is a classical molecule which can trigger endoplasmic reticulum (ER) stress and activate XBP1 splicing. When we treated MCF7-TAMR cells with thapsigargin, the XBP1 splicing was induced. However, when we co-applied STF-080310 with thapsigargin, the splicing process of XBP1 was efficiently blocked (Figure $2 \mathrm{~b}, 2 \mathrm{c}$ ), indicating that STF-080310 efficiently inhibits XBP1 function. Indeed, when we treated MCF7-TAMR cells with tamoxifen or STF-080310 separately, the cell viability was not affected, but when the MCF7-TAMRcells were treated with tamoxifen and STF-080310 together, the cell viability was significantly reduced (Figure $2 \mathrm{~d}$ ). Taken together, these results demonstrate that the IRE1/XBP1 inhibitor STF-080310 can restore tamoxifen sensitivity in MCF7-TAMR cells.

\section{STF-080310 suppresses breast tumor progression in a murine breast cancer xenograft model together with tamoxifen}

To evaluate whether STF-080310 can suppress tumorigenesis in vivo, we used a murine breast cancer xenograft model by injecting the MCF7-TAMR cells 
a<smiles>O=S(=O)(/N=C/c1c(O)ccc2ccccc12)c1cccs1</smiles>

C
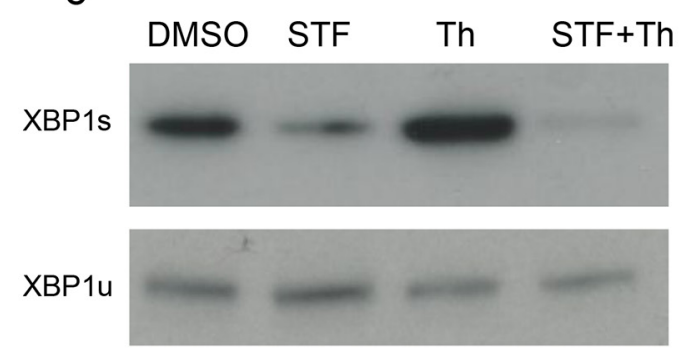

GAPDH

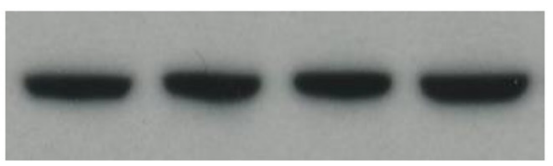

b

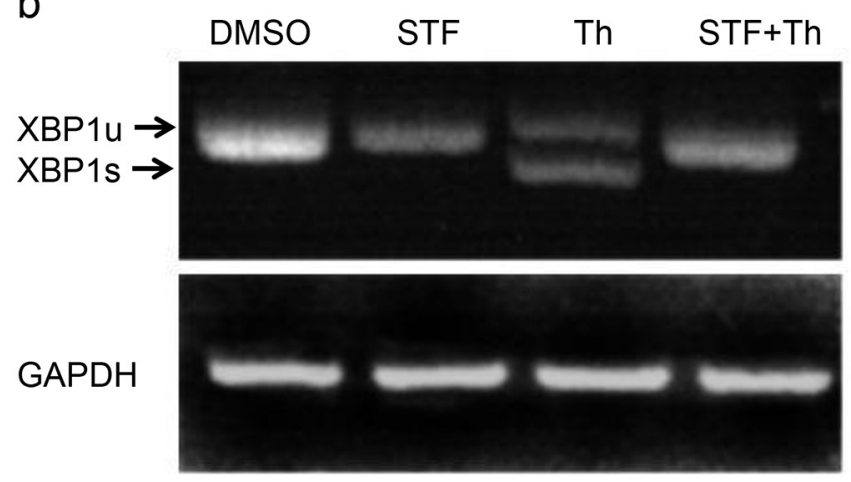

d

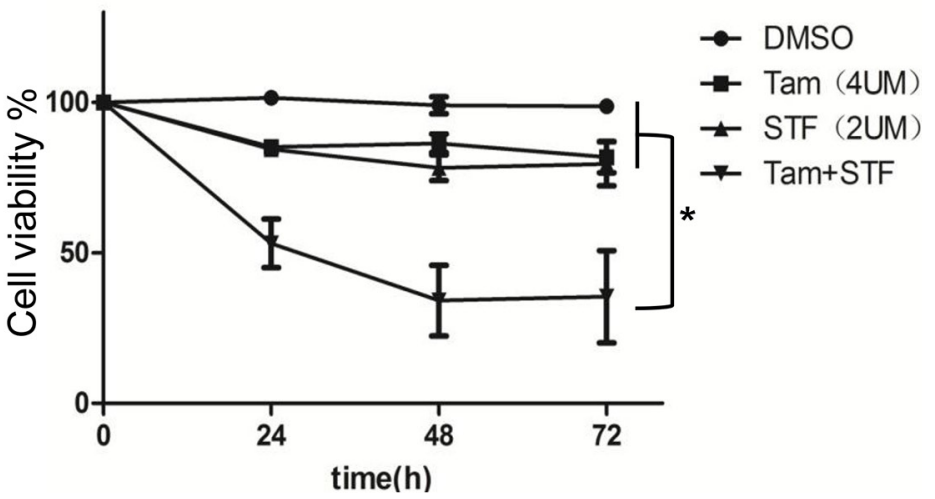

Figure 2: A novel IRE1/XBP1 inhibitor STF-080310 can block XBP1 splicing and restore tamoxifen sensitivity in vitro. a. Schematic chemical structure of STF-080310 (STF). b. RT-PCR analysis of XBP1 splicing in MCF7-TAMR cells. Thapsigargin(Th) is a classical chemical which can trigger UPR and XBP1 splicing. The upper band indicates XBP1u mRNA and the lower band indicates XBP1s mRNA. Note that STF can efficiently block XBP1 mRNA splicing even in Th-treated cells. c. Immunoblot analysis of XBP1s in DMSO, STF, Th and STF plus Th treatment in MCF7-TAMR cells, The XBP1u, and GAPDH are used as loading controls. d. Cell viability assay of MCF7-TAMR cells treated with STF-080310, tamoxifen, and dual treatment of STF and tamoxifen. Results from three different experiments are shown and suggest that STF can significantly restore tamoxifen sensitivity in vitro by inhibiting XBP1s activity. ${ }^{*} P<0.05$. (unpaired, two-sided $t$-test).

into the dorsal flank of female nude mice. When tumors reached approximately a size of $150 \mathrm{~mm}^{3}$, we divided the tumor-bearing mice into 4 different groups: control group (treated with DMSO); tamoxifen-treated group; STF-080310-treated group and tamoxifen plus STF080310 treated group. Each group was comprised of at least 7 mice and all the mice were treated for 3 weeks (Figure 3a). When we isolated the tumors from the four groups, the tamoxifen plus STF-080310-treated mice had smaller tumors compared to the control group and to the single drug treatment groups (Figure 3b). Moreover, tumor growth curve indicated that tamoxifen plus STF080310-treated mice showed significantly slower tumor progression compared to other 3 groups (Figure 3c), also the tumor weight from the dual treatment of tamoxifen and STF-080310 group were much less than other groups (Figure 3d). These results demonstrated that STF-080310 and tamoxifen had synergistic therapeutic effects on tamoxifen-resistant breast tumors in vivo.
Furthermore, we investigated the pathological and proliferation status of these tumors by H\&E and Ki67 staining, but no significant difference were detected among these groups (Supplementary Figure S2a, S2b), however, we found dramatically more Caspase 3 positive staining in the tamoxifen and STF-080310 treated tumors, suggesting more apoptotic cell death of tumor cells in this group (Figure 3e, 3f). Finally, we analyzed $\mathrm{XBP} 1 \mathrm{~s}$ expression levels in all the breast tumors by immunohistochemistry and found that the XBP1s expression was significantly inhibited in both groups treated with STF-080310 alone or with STF080310 and tamoxifen. (Supplementary Figure S3), which indicated that STF-080310 could efficiently inhibit XBP1s' expression in vivo. Taken all together, STF080310 can significantly reinstate tamoxifen sensitivity in vivo by inhibiting XBP1s function and thus can be used together with tamoxifen to efficiently delay breast tumor progression. 

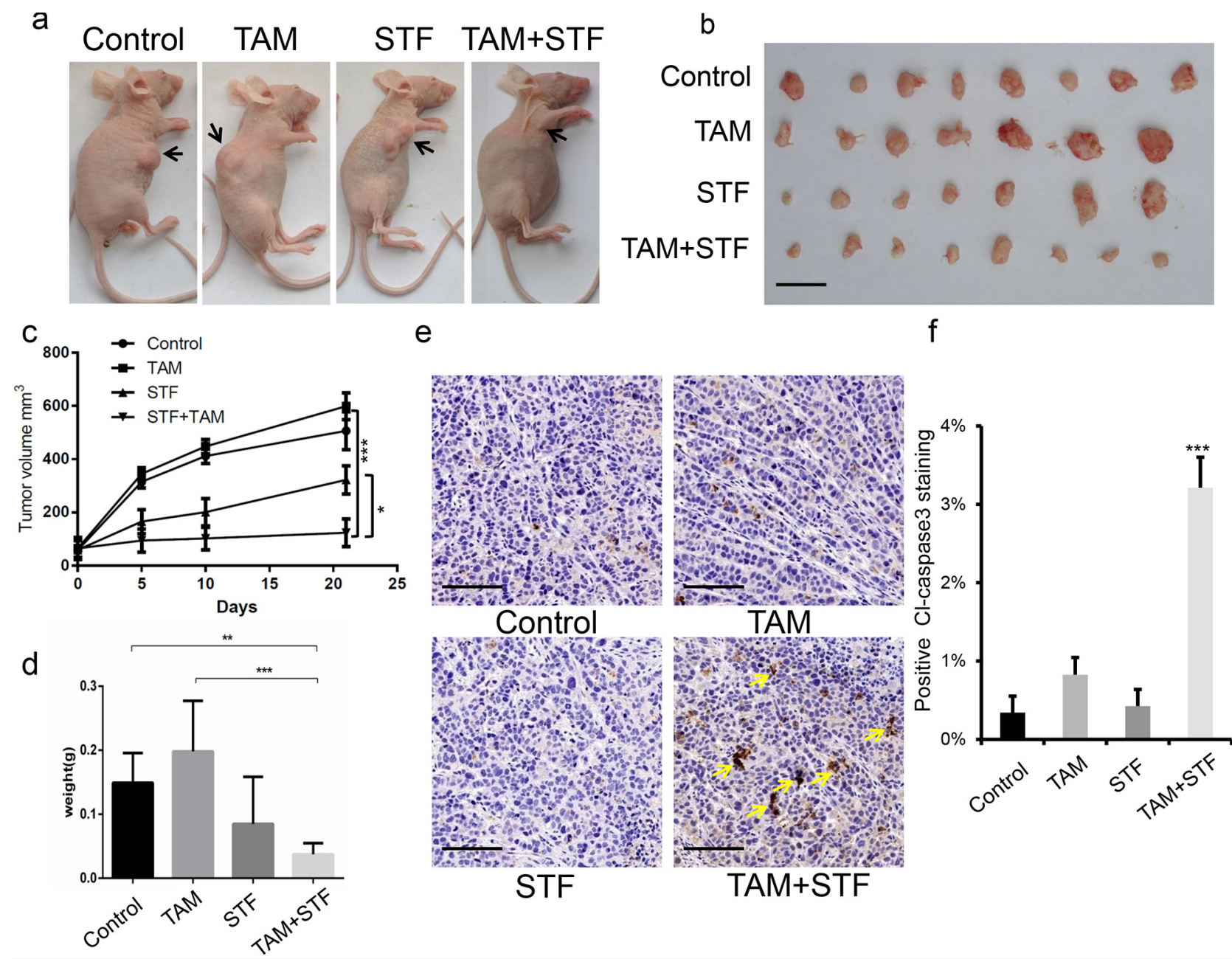

Control

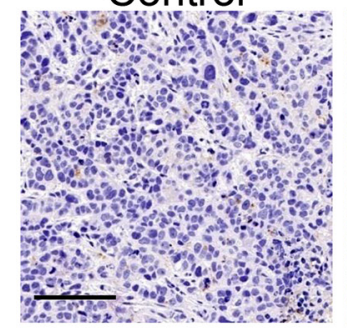

STF

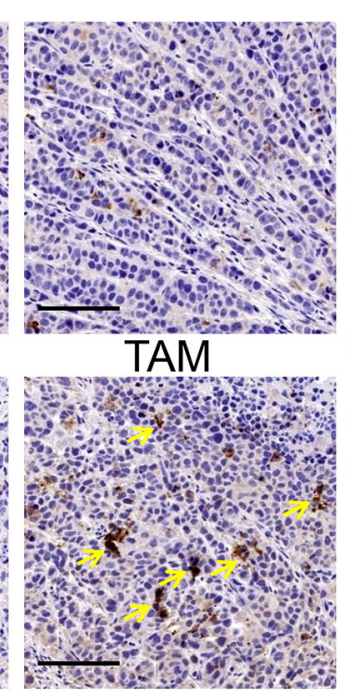

TAM+STF

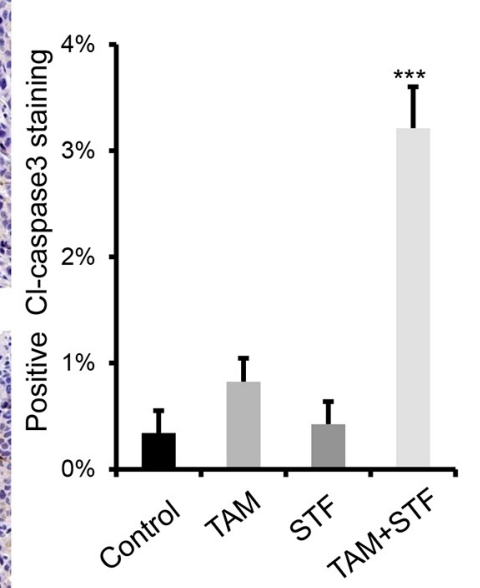

Figure 3: STF-080310 has a synergistic therapeutic effect with tamoxifen in a murine xenograft breast tumor model. a. Representative images of nude mice 21 days after injected with MCF7-TAMR cells of control group; tamoxifen-treated group (TAM); STF-080310-treated group (STF) and tamoxifen- plus STF-080310-treated group (TAM+STF). b. At least 7 mice per group were sacrificed to analyze tumor progression. Compared to the other groups, the TAM+STF treated mice had significantly smaller tumors. Scale bars, $1 \mathrm{~cm}$. c. Tumor growth curve of control group, TAM group, STF group and TAM+STF group. Tumors' sizes were determined 5 days, 10 days and 21 days after injecting MCF7-TAMR cells into nude mice. ${ }^{*} P<0.05$; $* * * P<0.001$ (Wald's test). d. Tumor weights were measured and results are shown as mean $( \pm$ s.e.m). The TAM $+\mathrm{STF}$ treatment significantly decreases tumor weight compare to control group and to the two single treatment groups, suggesting a synergistic effect of STF and TAM in tamoxifen-resistant breast tumor therapy in vivo. $* * P<0.01 ; * * * P<0.001$. (unpaired, two-sided $t$-test). e. Immunohistochemistry analysis of Caspase 3 in breast tumor tissue in all 4 groups. Yellow arrows indicate positive Caspase 3 staining. Scale bars, $50 \mu \mathrm{m}$. f. Positive Caspase 3 staining of 4 treatment groups are shown as mean percentages ( \pm s.e.m.) of (e). ${ }^{* * *} P<0.001$. (unpaired, two-sided $t$-test).

\section{Elevated XBP1s expression is highly correlated with poor survival in $\mathrm{ER}^{+}$breast cancer patients}

Over 170 patients' samples were collected for immunohistochemistry analysis for XBP1s expression. The patients had been categorized into different sub groups according to their various characteristics. We established standard protocols to define high and low expression of XBP1s (see details in Materials and Method), and representative images of high and low XBP1s expression levels are shown in Figure 4a. Furthermore, we investigated the correlation between XBP1s expression and overall survival. The results indicated that for the high XBP1s expression patients, the survival ratio was only 46.7\%. Conversely, for low XBP1s expression patients the survival ratio was $75 \%$, which was significantly higher than high expression patients (Figure 4b). Moreover, we analyzed the correlation of XBP1s expression and survival both in $\mathrm{ER}^{+}$and $\mathrm{ER}^{-}$subgroups respectively, and found that XBP1s expression was only associated with overall survival in the $\mathrm{ER}^{+}$group but not in the $\mathrm{ER}^{-}$group (Figure 4c, 4d). 

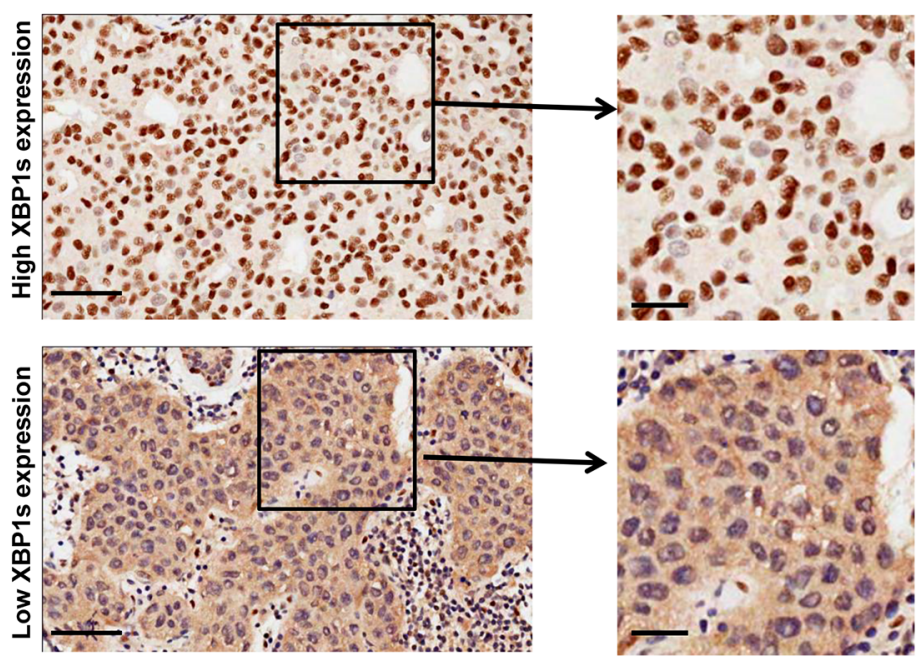

$\mathrm{b}$

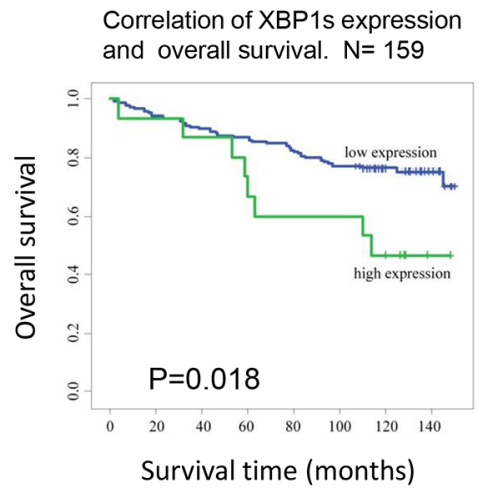

C

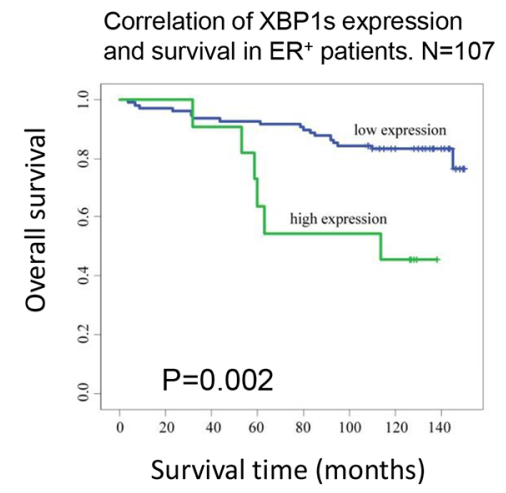

d

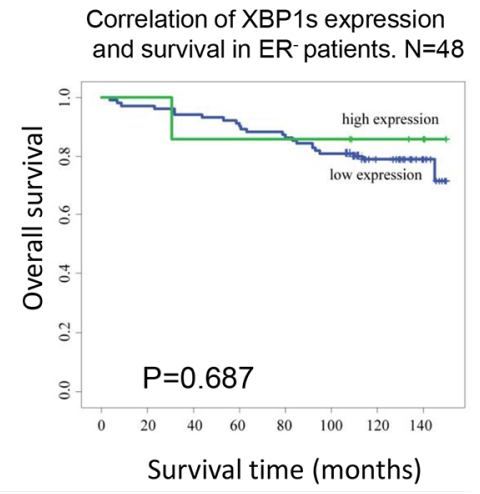

Figure 4: XBP1s expression is highly correlated with overall survival of ER+ breast cancer patients. a. Representative images of high and low XBP1s expression level in clinical patients' samples. Scale bars, left panel, $100 \mu \mathrm{m}$, right panel, $20 \mu \mathrm{m}$. b, c, d. Correlation of XBP1s expression level and overall survival in all patients (b), $\mathrm{ER}^{+}$patients (c) and $\mathrm{ER}^{-}$patients (d). $P<0.05$ is considered as significant.

\section{XBP1 is not an independent prognostic factor in $\mathbf{E R}^{+}$and $\mathbf{E R}^{-}$groups}

All the patients were categorized according to their pathological grade, clinical stage, as well as their ER, PR and HER2 status (Figure 5). We analyzed different prognostic factors and found that only in $\mathrm{ER}^{+}$groups did XBP1s expression as well as pathological levels, tumor size, $\mathrm{T}$ stage, $\mathrm{N}$ stage, TNM stage significantly affect overall survival. However, in the $\mathrm{ER}^{-}$group, all these factors had no correlation with overall survival by Logrank test (Table 1). Multivariate Cox regression analysis in $\mathrm{ER}^{+}$and $\mathrm{ER}^{-}$groups are displayed in Table 2. In the $\mathrm{ER}^{+}$ group, XBP1s expression had no statistically significant contribution to prognosis $(P=0.074)$ although the relative risk was obviously high $(2.539,95 \% \mathrm{CI} 0.931-7.059)$. The only factor that had statistical significance in this group was tumor size $(P=0.039)$, with a RR of $2.943(95 \% \mathrm{CI}$ 1.056-8.200). However, none of these factors displayed statistically significance in prognostic predicting in the
$\mathrm{ER}^{-}$group. Overall, it is concluded that XBP1s expression cannot be used as an independent prognostic factor.

\section{DISCUSSION}

Endocrine therapy is the most efficient systemic therapy for estrogen receptor positive breast cancer; however, many $\mathrm{ER}^{+}$breast cancer patients can acquire resistance to endocrine therapy. Previous studies have shown that this resistance is associated with the UPR response and elevated signal transduction pathways such as the EGFR/MAPK, PI3K/AKT/mTOR pathways [15]. Alternative treatments include applying different endocrine drugs such as anastrozole and fulvestrant; or combinational endocrine therapy with small molecular inhibitors such as gefitinib, lapatinib and everolimus [16]. Clinical studies have shown that aromatase inhibitors (AIs) can significantly increase the therapeutic efficiency of postmenopausal breast cancer patients who have tamoxifen resistance. Even among those patients who 


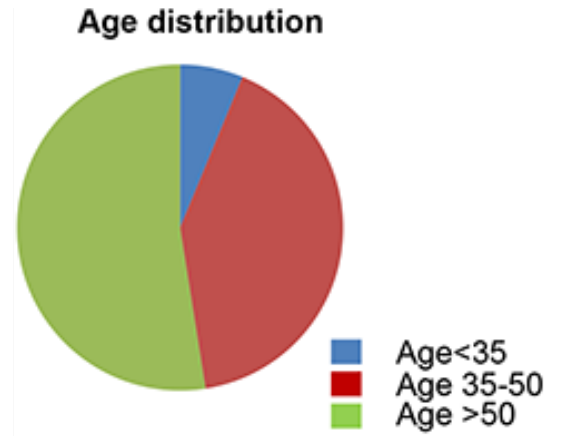

Pathology distribution

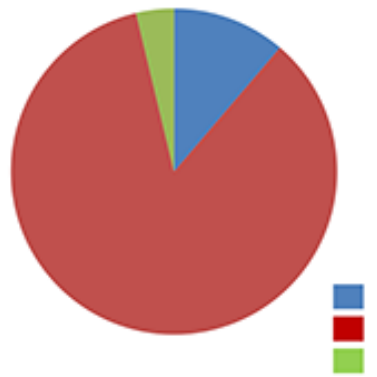

Grade 1

Grade 3
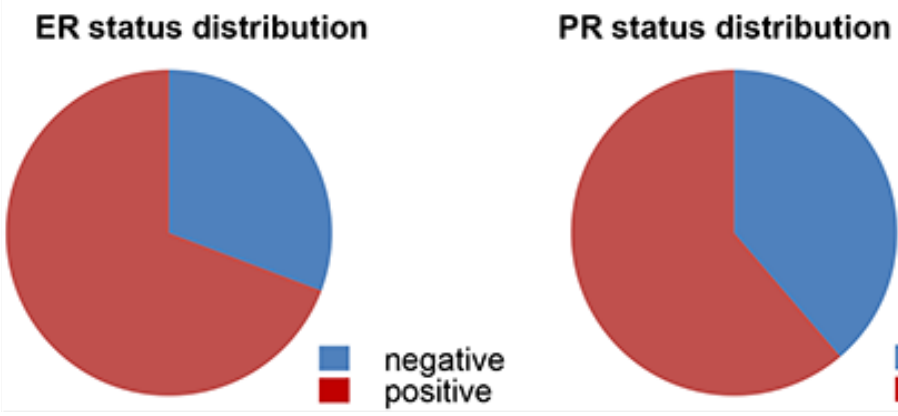

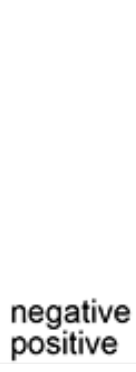

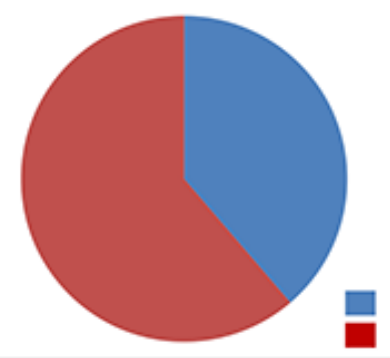

Clinical stage distribution

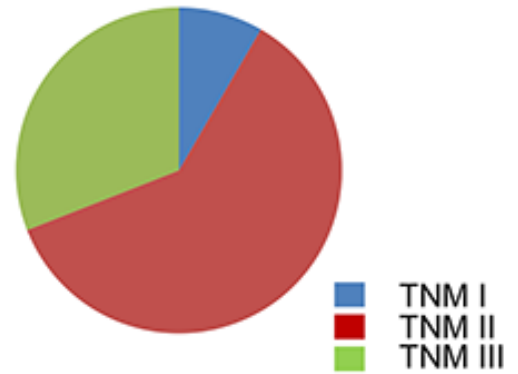

HER2 status distribution

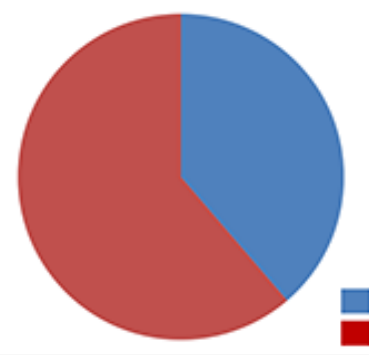

negative

negative positive

Figure 5: Characterization of patients. Patients are categorized with respect to age, pathology grade, clinical stage classification, ER status, PR status and HER2 status.

got no therapeutic effects with AIs, $30 \%$ of them can benefit with subsequent usage of fulvestrant $[17,18]$. It is also reported that mTOR inhibitors in combination with exemestane can improve progression-free survival in postmenopausal breast cancer patients. [15] In vitro studies show that mTOR inhibitors combined with tamoxifen can increase toxicity by killing up to $56 \%$ more $\mathrm{ER}^{+}$breast tumor cells than the single drug treatment [19]. Despite these inspiring data, clinical trials evaluating the combination of endocrine therapy and trastuzumab or the TKIs, like gefitinib, erlotinib and lapatinib, have produced various results. $[20,21]$ Nevertheless, tamoxifen is still the most important endocrine therapy for premenopausal breast cancer patients, therefore it is important and urgent to investigate the mechanisms involved in breast tumor cell resistance to tamoxifen and to establish new treatment strategies.

Human X-box binding protein-1 (XBP1) is an alternatively spliced transcription factor that participates in a stress-signaling pathway to protect cells from damage. It has also been reported that XBP1 is involved in the unfolded protein response (UPR) and ER stress response under the control of IRE1. Clarke et.al found that overexpression of the spliced variant of the gene in estrogen receptor-positive breast cancer cells led to reduced sensitivity to tamoxifen and faslodex. Subsequent studies reported that $\mathrm{XBP} 1 \mathrm{~s}$ expression in $\mathrm{ER}^{+}$breast tumors correlated with poor clinical responsiveness to tamoxifen, however, the underlying signaling mechanisms affected by XBP1s, as well as the effects of splicing on antiestrogen resistance, remain unclear. It is hypothesized that $\mathrm{XBP} 1 \mathrm{~s}$ mediates anti-estrogen resistance in part through regulating nuclear factor kappa $\mathrm{B}$ (NF-kappaB) signaling. Overexpression of XBP1 in MCF7 and LCC1 antiestrogen-sensitive breast cancer cells resulted primarily in an increase in XBP1s and an induction of RelA expression at both the mRNA and protein levels [22] Chen et.al reported that XBP1 was activated in triple negative breast cancer, a highly aggressive malignant subtype of breast cancer. They found that deletion of XBP1inhibited both tumor growth and tumor relapse as well as decreased the $\mathrm{CD} 44^{\text {high }} \mathrm{CD} 24^{\text {low }}$ cell population by inhibiting the HIF pathway. [23]

In our study, we established a MCF7-TAMR cell line by long-term, low concentration exposure of MCF-7 cell to tamoxifen. Cell viability assays showed that the MCF7-TAMR cells' sensitivity to tamoxifen was reduced by $1.7 \sim 2.4$ fold compare to control cells; this was also confirmed by morphological analysis. The method of low concentration tamoxifen exposure to induce cell resistance to tamoxifen is a reasonable and efficient way of studying drug resistance in vitro, which essentially mimics the reallife clinical situation drug resistance occurs, as most drug resistance is induced by long term drug usage.

We next investigated the expression levels of XBP1 and its upstream regulator, IRE1, in the MCF7-TAMR cell 
Table 1: Kaplan-meier survival analysis grouped by ER status respective to XBP1s expression and other clinicalpathologic parameters

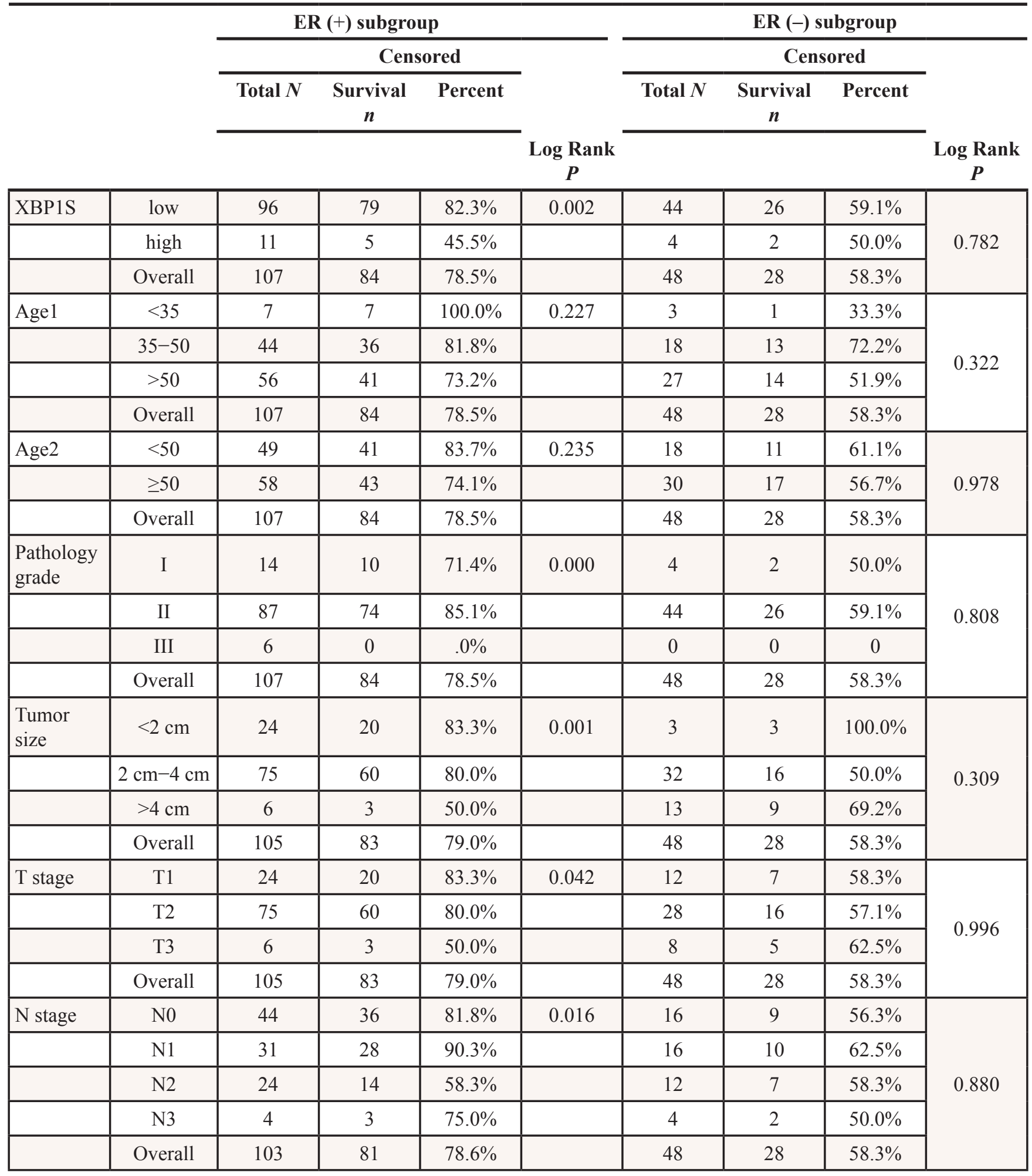

(Continued) 


\begin{tabular}{|c|c|c|c|c|c|c|c|c|c|}
\hline & & \multicolumn{3}{|c|}{ ER (+) subgroup } & & \multicolumn{4}{|c|}{ ER (-) subgroup } \\
\hline & & \multirow{3}{*}{ Total $N$} & \multicolumn{2}{|c|}{ Censored } & \multirow[b]{3}{*}{$\begin{array}{c}\text { Log Rank } \\
P \\
\end{array}$} & \multicolumn{3}{|c|}{ Censored } & \multirow[b]{3}{*}{$\begin{array}{c}\text { Log Rank } \\
P \\
\end{array}$} \\
\hline & & & $\begin{array}{c}\text { Survival } \\
n\end{array}$ & Percent & & Total $N$ & $\begin{array}{c}\text { Survival } \\
n\end{array}$ & Percent & \\
\hline & & & & & & & & & \\
\hline \multirow[t]{4}{*}{$\begin{array}{l}\text { TNM } \\
\text { stage }\end{array}$} & TNM1 & 9 & 7 & $77.8 \%$ & 0.012 & 4 & 4 & $100.0 \%$ & \multirow{4}{*}{0.305} \\
\hline & TNM2 & 64 & 56 & $87.5 \%$ & & 26 & 14 & $53.8 \%$ & \\
\hline & TNM3 & 29 & 18 & $62.1 \%$ & & 18 & 10 & $55.6 \%$ & \\
\hline & Overall & 102 & 81 & $79.4 \%$ & & 48 & 28 & $58.3 \%$ & \\
\hline \multirow[t]{3}{*}{ PR } & - & 18 & 13 & $72.2 \%$ & 0.441 & 42 & 24 & $57.1 \%$ & \multirow{3}{*}{0.447} \\
\hline & + & 88 & 70 & $79.5 \%$ & & 4 & 3 & $75.0 \%$ & \\
\hline & Overall & 106 & 83 & $78.3 \%$ & & 46 & 27 & $58.7 \%$ & \\
\hline \multirow[t]{3}{*}{ HER-2 } & - & 77 & 60 & $77.9 \%$ & 0.935 & 26 & 14 & $53.8 \%$ & \multirow{3}{*}{0.470} \\
\hline & + & 30 & 24 & $80.0 \%$ & & 22 & 14 & $63.6 \%$ & \\
\hline & Overall & 107 & 84 & $78.5 \%$ & & 48 & 28 & $58.3 \%$ & \\
\hline \multirow[t]{3}{*}{$\begin{array}{l}\text { HER- } \\
\text { 2(FISH) }\end{array}$} & - & 81 & 63 & $77.8 \%$ & 0.923 & 28 & 14 & $50.0 \%$ & \multirow{3}{*}{0.093} \\
\hline & + & 14 & 11 & $78.6 \%$ & & 4 & 4 & $100.0 \%$ & \\
\hline & Overall & 95 & 74 & $77.9 \%$ & & 32 & 18 & $56.3 \%$ & \\
\hline
\end{tabular}

Table 2: Multivariate Cox regression analysis in $\mathbf{E R}^{+}$and $\mathbf{E R}^{-}$groups

\begin{tabular}{|c|c|c|c|c|c|c|c|c|c|c|c|c|c|}
\hline & \multicolumn{6}{|c|}{ ER (+) subgroup } & \multicolumn{7}{|c|}{ ER (-) subgroup } \\
\hline & \multirow[b]{2}{*}{$\mathrm{CC}$} & \multirow[b]{2}{*}{ SE } & \multirow[b]{2}{*}{$\mathbf{P}$} & \multirow[b]{2}{*}{$\mathbf{R R}$} & \multicolumn{2}{|c|}{$95.0 \%$ CI } & & \multirow[b]{2}{*}{$\mathrm{CC}$} & \multirow[b]{2}{*}{ SE } & \multirow[b]{2}{*}{$\mathbf{P}$} & \multirow[b]{2}{*}{$\mathbf{R R}$} & \multicolumn{2}{|c|}{$95.0 \% \mathrm{CI}$} \\
\hline & & & & & lower & upper & & & & & & lower & upper \\
\hline XBPISn & .932 & .522 & .074 & 2.539 & .913 & 7.059 & XBPISn & .730 & .421 & .083 & 2.074 & .909 & 4.736 \\
\hline T stage & -.035 & .608 & .954 & .966 & .294 & 3.177 & Pathology & .621 & .537 & .247 & 1.862 & .650 & 5.331 \\
\hline $\mathrm{N}$ stage & .206 & .442 & .641 & 1.228 & .517 & 2.920 & $\mathrm{~N}$ stage & -079 & .311 & .799 & .924 & .502 & 1.701 \\
\hline $\begin{array}{l}\text { TNM } \\
\text { stage }\end{array}$ & .439 & .802 & .584 & 1.551 & .322 & 7.466 & $\begin{array}{l}\text { TNM } \\
\text { stage }\end{array}$ & .752 & .529 & .155 & 2.122 & .752 & 5.990 \\
\hline $\begin{array}{l}\text { Tumor } \\
\text { size }\end{array}$ & 1.079 & .523 & .039 & 2.943 & 1.056 & 8.200 & ER status & -.409 & .447 & .360 & .664 & .276 & 1.596 \\
\hline Pathology & .937 & .544 & .085 & 2.551 & .878 & 7.414 & PR status & -.607 & .450 & .177 & .545 & .226 & 1.316 \\
\hline
\end{tabular}

lines, and showed that the expression level of both XBP1s and p-IRE1 were increased significantly. Importantly, this enhanced expression occurred shortly after tamoxifen was given and in a concentration-dependent manner. Although there may be other pathways affected in the MCF7-TAMRcells, we conclude that the up-regulation of IRE1-XBP1 is at least partially associated with the reduced sensitivity to tamoxifen, and that the UPR and ER stress responses are activated in MCF7-TAMR cells. Indeed, we compared gene expression profiles between
MCF7-TAMR cells and control cells using a next generation sequencing approach and a new bioinformatics model, the result showed $1215 \mathrm{mRNA}$ and 513 small RNA transcripts were changed which could be clustered into ER functions, cell cycle regulation, transcription/translation, and mitochondrial dysfunction.

To validate our findings, we next used a novel IRE1-XBP1 inhibitor, STF-083010, to treat the MCF7TAMRcells. STF083010 is an inhibitor of IRE1 $\alpha$ endonuclease activity; which can block endogenous XBP1 
mRNA splicing and displays cytostatic and cytotoxic effects in $\mathrm{CD}_{138^{+}}$multiple myeloma (MM) cells in vitro [14], STF083010 is also reported to inhibit bortezomibinduced XBP1 activity in myeloma xenografts in vivo but does not alter IRE1 $\alpha$ kinase activity [24]. Our in vitro experiments showed that STF-083010 could specifically inhibit XBP1 splicing and increased the sensitivity of the MCF7-TAMRcells to tamoxifen by up to $60 \%$. We also injected MCF7-TAMRcells into nude mice to study STF083010 function in vivo. Not surprisingly, the combinatory treatment of STF-083010 and tamoxifen could reduce tumor weight and tumor diameter by up to $75 \%$ and $38.3 \%$ respectively when compared to single-drug treatment groups. As described previously [13, 14], STF-083010is a novel IRE1-XBP1 inhibitor which inhibits the RNase activity of IRE1 but without affects its kinase functions, thereby specifically regulating downstreamXBP1s expression while greatly minimizing undesired sideeffects. We believe that the decreased XBP1s expression level induced by STF-083010 somehow restored the tumor sensitivity to tamoxifen, however, we cannotexclude other factors which might contribute to this striking therapeutic result, and the detailed mechanism of how STF-083010 reestablishes the sensitivity of resistant tumors to tamoxifen in vivo needs to be further investigated.

As XBP1s has been implied to play a key role in drug resistance in certain subtypes of breast cancers [23], we systematically reviewed breast cancer patients' samples regarding XBP1s expression. Intriguingly, we found thatXBP1s expression was correlated with overall survival not only in the $\mathrm{ER}^{+}$patients, but also among all breast cancer patients when we pooled them together. Our data demonstrates that high XBP1s expression levels lead to poor survival after endocrine therapy, which suggests that $\mathrm{XBP} 1 \mathrm{~s}$ could be considered as a prognosis factor and contribute to drug resistance in the clinic. Therefore, small molecular inhibitors such as STF083010, which targets IRE1-XBP1, may have potential therapeutic effects for endocrine therapy resistant patients.

In essence, IRE1-XBP1 plays an essential role in the UPR and ER stress responses, which have been implicated in the development of drug resistance. Our data suggests that a combination of IRE1-XBP1 inhibitors and endocrine therapy would be an alternative strategy for breast cancer therapy in the future.

\section{MATERIALS AND METHODS}

\section{MCF-7 cell cultures, T47D cell cultures and establishment of tamoxifen-resistant cell lines}

The original MCF-7 and T47D breast cancer cells were purchased from ATCC. Both cells were cultured in DMEM supplemented with 10\% fetal bovine serum (Gibco, Life Technology) and 1\% penicillin-streptomycin (standard medium) at $37^{\circ} \mathrm{C}$ and $5 \% \mathrm{CO} 2$. To develop tamoxifen-resistant cells, the MCF-7 cells were cultured in the same conditions supplemented with $1 \mu \mathrm{M} 4-\mathrm{OH}$ tamoxifen (Sigma Aldrich) for 30 days and the T47D cells were incubated with $10 \mu \mathrm{M} 4-\mathrm{OH}$ tamoxifen for 30 days respectively.

\section{Quantitative RT-PCR}

Total RNA was prepared from control and tamoxifen-resistant MCF-7 cells using the RNeasy Mini Kit (Qiagen) in accordance with the manufacturer's instructions. Total RNA ( $1 \mu \mathrm{g})$ was subjected to reverse transcription using random hexamers (Roche) and SuperscriptII (Invitrogen) followed by quantitative PCR analysis. The following primers were used:

XBP1 spliced: 5'-GGTCTGCTGAGTCCG CAGCAGG-3' (forward) and 5'GGGCTTGGTATATATGTGG-3' (reverse)

XBP1 total (XBP1 spliced+XBP1 unspliced): 5'CGGAAGCCAAGGGGAATGAA-3' (forward) and 5'GTCCAGAATGCCCAACAGGA-3' (reverse)

GAPDH: 5'- GAAGGTGAAGGTCGGAGTC-3' (forward) and 5'- GAAGATGGTGATGGGATTTC-3' (reverse).

\section{Western blot analysis}

Approximately $2 \times 10^{6}$ cells of each cell line were prepared overnight in 6-well plates. The next day, the supernatant was discarded and the cells were washed twice with PBS. Next the cells were lysed with appropriate RIPA buffer containing 1\%PMSF, placed $20 \mathrm{~min}$ on ice and scraped using cell scraper. After 20 min incubation, cells/lysates were collected and transferred into tubes and centrifuged at $12,000 \mathrm{~g}$ at $4^{\circ} \mathrm{C}$ for $10 \mathrm{~min}$. The supernatant was pipetted into new tubes and the protein concentrations were determined by the BCA method (Thermo Scientific). Equal amount of protein (40 ug) of lysates were used for electrophoresis using Nu-Page 10\% Bis-Tris gels and blotted onto nitrocellulose membranes. The membranes were washed briefly in TBS $/ 0.1 \%$ Tween-20 (TBST), $\mathrm{pH} 7.4$, and blocked in $5 \%$ milk or $5 \%$ BSA (diluted in TBST) for $1 \mathrm{~h}$. The membranes were thenincubated with the following antibodies: XBP1s primary antibody (1:500; Abcam, 198999), XBP1u (1:1000, Abcam, abab37152), p-IRE1 (1:1000; Abcam, ab104157), IRE1 (1:1000; Cell Signaling, \#3294), p-AKT (1:1000; Cell signaling, \#9611), AKT (1:4000; Cell Signaling,\#2944), Caspase3 (1:2000, Cell signaling,\#9665), GADPH (1:5000; Cell Signaling, \#5174) diluted in blocking solution overnight. Blots were then washed with TBST for $5 \mathrm{~min}$ three times and incubated in corresponding secondary antibody (1:20000; Cell Signaling, \#7071, \#7072) for $1 \mathrm{~h}$. The membranes were processed with ECL-plus (Pierce, 32132) and then exposed on films in a dark room and the films were scanned by software Quantity One (Bio-Rad) and quantified by densitometry. 


\section{Cell viability assay}

Cell viabilities were determined by the MTT assay. Cells were seeded into 96-well culture plates with $0.5 \%$ fetus serum medium and started to adhere after $2-4$ hours. Then the cells were treated with different conditions. After the treatment, cells were incubated with MTT working solution (Roche). The cell viabilities were determined by acquiring the OD value at $540 \mathrm{~nm}$ after incubation 4-6 hours in MTT. The relative viability was determined by OD value of experiment group/control group $\times 100 \%$.

\section{Immunohistochemistry}

Immunohistochemical analysis for XBP1s in breast tumor mice or patients was performed in a standard protocol. Briefly, $2 \mu \mathrm{m}$ sections were obtained from formalin fixed and paraffin embedded tumor samples, sections were dehydrated and antigenic epitopes retrieved using a $10-\mathrm{mM}$ citrate buffer and microwaving for 10 minutes. Specimens were stained with XBP1s antibody (1:100, abcam, ab37152), primary antibody staining was detected by peroxidase conjugated anti-rabbit IgG according to manufacturer's instructions (Roche). The ER, PR, and Her-2/neu staining were previously performed and recorded after initial surgery was done. The immunohistochemical expression of XBP1s was examined by light microscopy through calculating 1000 cells per 5 sights. The percentage of positive cells, as the extent of immunostaining, was quantified under the microscope and classified into five groups. $0:<5 \%$ positive cells; $1: 5 \%$ to $24 \%$ positive cells; $2: 25 \%$ to $49 \%$ positive cells; $3: 50 \%$ to $74 \%$ positive cells and $4:>75 \%$ positive cells. Intensity was scored as 0 for absence of staining, 1 for weak, 2 for moderate, and 3 for strong staining. The score of the intensity plus the percentage of positive staining were used to define expression levels. $0-1$ : negative; $2-3$ : little positive $(+1) ; 4-5$ : moderately positive (+2); 6-7: strongly positive $(+3)$. Lastly, a total score calculated by the product of staining intensity and positive percentage was used to divide all specimens into two groups: a low-expression group ( $0-5$ scores $)$ and a high-expression group (6-12 scores). Immunhistochemical staining was done using an automatic staining machine (Leica Bond3) or manually processed. Sections were dehydrated and antigenic epitopes were retrieved using a 10-mM citrate buffer and microwaving for $10 \mathrm{~min}$. Specimen were then incubated with rabbit anti-Ki67 (Novocastra, 1:200), anticleaved caspase-3 (Cell Signaling, 9661, 1:200). Primary Ab staining was detected by peroxidase conjugated anti-rabbit $\operatorname{IgG}$ (DAKO, P0448, 1:500). Positive cells were counted on 20 randomly chosen tumor areas at $\times 400$ magnifications in a double blinded fashion.

\section{Tumor growth model in nude mice}

All mice were maintained according to the ethical animal license protocol complying with the Chinese law, and all animal studies were approved by the Huazhong Science and Technology University (HUST) Institutional Animal Care and Use Committee. Briefly, $4 * 10^{6}$ tamoxifenresistant MCF-7 cells were injected subcutaneously into the flankof 4-week-old female nude mice (Animal experiment center of Tongji Medical College, HUST, WuHan, China). 4 days later, all the mice were divided into four groups randomly and treated with: 1) DMSO, 2) TAM 100ug/ kg per day, 3) STF-083010 30mg/kg per week, 4) TAM combined with STF- 083010 by intraperitoneal injection. Three weeks after injection, animals were killed and the four groups of tumors were examined. Tumor growth was evaluated by measuring tumor weight.

\section{Patients and samples}

A total of 170 invasive breast cancer patients who underwent surgery at hospitalsof Jiangsu, Zhejiang and Shanghai that cooperated with National Engineering Center for Biochip at Shanghai during 2001-2004 were selected. 164 patients remained when six were discarded from the analysis due to lack of follow-up or lack of data. The age of patients ranged from 29 to 82 years old. Clinic-pathological characteristics are presented in Table 1. ER or PR-positive breast cancers were supposed to use tamoxifen as endocrine therapy under standard treatment.

\section{Statistical analyses}

All statistical analyses were performed using SPSS16.0 software and the detail methods were indicated in figure legend. Survival curves were calculated using the Kaplan-Meier method, with the significance evaluated using the Mantel-Cox long-rank test. The prognostic significance of the parameters was assessed using the Cox proportional hazards model with overall survival as an end point. A multivariate analysis was performed using Cox model; previously identified prognostic factors in breast cancer were included in the model. The relations of XBP1s expression and clinic-pathological parameters were measured using the nonparametric KrusKal-Wallis and Mann-Whitney methods, using Spearman's correlation analysis. $p<0.05$ was considered statistically significant. Correlations with numerical variables were analyzed by Mann-Whitney $U$ test.

\section{ACKNOWLEDGMENTS AND FUNDING}

We thank everyone in our department for discussion and suggestions; Dr. Shuan Rao helped with manuscript preparation. This work was funded by National Natural Science Foundation of China (NSFC81202094).

\section{CONFLICTS OF INTEREST}

We declare no competing financial interest. 


\section{REFERENCES}

1. Viedma-Rodríguez R, Baiza-Gutman L, Salamanca-Gómez F, Diaz-Zaragoza M, Martínez-Hernández G, Ruiz EsparzaGarrido R, Velázquez-Flores MA, Arenas-Aranda D. Mechanisms associated with resistance to tamoxifen in estrogenreceptor-positive breast cancer. Oncol Rep. 2014; 32:3-15.

2. Kim I, Xu W, Reed JC. Cell death and endoplasmic reticulum stress: disease relevance and therapeutic opportunities. Nat Rev Drug Discov. 2008; 7:1013-30.

3. Clarke R, Cook KL, Hu R, Facey CO, Tavassoly I, Schwartz JL, Baumann WT, Tyson JJ, Xuan J, Wang Y, Wärri A, Shajahan AN. Endoplasmic reticulum stress, the unfolded protein response, autophagy, and the integrated regulation of breast cancer cell fate. Cancer Res. 2012; 72:1321-31.

4. Lin JH, Li H, Yasumura D, Cohen HR, Zhang C, Panning B, Shokat KM, Lavail MM, Walter P. IRE1 signaling affects cell fate during the unfolded protein response. Science. 2007; 318:944-9.

5. Han D, Lerner AG, Vande Walle L, Upton JP, Xu W, Hagen A, Backes BJ, Oakes SA, Papa FR. IRE1 $\alpha$ Kinase Activation Modes Control Alternate Endoribonuclease Outputs to Determine Divergent Cell Fates. Cell. 2009; 3:562-575.

6. Yoshida H, Matsui T, Yamamoto A, Okada T, Mori K. XBP1 mRNA is induced by ATF6 and spliced by IRE1 in response to ER stress to produce a highly active transcription factor. Cell. 2001; Dec 28;107:881-91.

7. Ding L, Yan J, Zhu J, Zhong H, Lu Q, Wang Z, Huang C, Ye Q. Ligand-independent activation of estrogen receptor a by XBP-1. Nucleic Acids Res. 2003; 31:5266-74.

8. Fang Y, Yan J, Ding L, Liu Y, Zhu J, Huang C, Zhao H, Lu Q, Zhang X, Yang X, Ye Q. XBP-1 increases ERa transcriptional activity through regulation of large-scale chromatin unfolding. Biochem Biophys Res Commun. 2004; 323:269-74.

9. Gomez BP, Riggins RB, Shajahan AN, Klimach U, Wang A, Crawford AC, Zhu Y, Zwart A, Wang M, Clarke R. Human X-box binding protein-1 confers both estrogen independence and antiestrogen resistance in breast cancer cell lines. FASED J. 2007; 21:4013-4027.

10. Davies MP, Barraclough DL, Stewart C, Joyce KA, Eccles RM, Barraclough R, Rudland PS, Sibson DR. Expression and splicing of the unfolded protein response gene XBP-1 are significantly associated with clinical outcome of endocrine-treated breast cancer. Int J Cancer. 2008; 123:85-88.

11. Zhu Y, Singh B, Hewitt S, Liu A, Gomez B, Wang A, Clarke R. Expression patterns among interferon regulatory factor-1, human $\mathrm{X}$ box binding protein-1, NFkB, nucleophosmin, estrogen receptor-a and progesterone receptor proteins in breast cancer tissue microarrays. Int J Oncol. 2006; 28:67-76.

12. Clarke R, Shajahan AN, Wang Y, Tyson JJ, Riggins RB, Weiner LM, Bauman WT, Xuan J, Zhang B, Facey C, Aiyer H, Cook K, Hickman FE, et al. Endoplasmic reticulum stress, the unfolded protein response, and gene network modeling in antiestrogen resistant breast cancer. Horm Mol Biol Clin Investig. 2011; 5:35-44.

13. Jha BK, Polyakova I, Kessler P, Dong B, Dickerman B, Sen GC, Silverman RH. Inhibition of RNase, L, and RNAdependent protein kinase (PKR) by sunitinib impairs antiviral innate immunity. J Biol Chem. 2011; 286:26319-26.

14. Papandreou I, Denko NC, Olson M, Van Melckebeke H, Lust S, Tam A, Solow-Cordero DE, Bouley DM, Offner F, Niwa M, Koong AC. Identification of an Ire1alpha endonuclease specific inhibitor with cytotoxic activity against human multiple myeloma. Blood. 2011; 117:1311-4.

15. Shou J, Massarweh S, Osborne CK, Wakeling AE, Ali S, Weiss H, et al. Mechanisms of Tamoxifen Resistance: Increased Estrogen Receptor-HER2/neu Cross-Talk in ER/ HER2-Positive Breast Cancer. JNCI Journal of the National Cancer Institute. 2004; 96:926-35.

16. Miller TW, Balko JM, Arteaga CL. Phosphatidylinositol 3-kinase and antiestrogen resistance in breast cancer. Journal of clinical oncology : official journal of the American Society of Clinical Oncology. 2011; 29:4452-61.

17. Johnston S, Cheung K. Fulvestrant-a novel endocrine therapy for breast cancer. Current medicinal chemistry. 2010; 17:902-14.

18. Cheung KL, Owers R, Robertson JF. Endocrine response after prior treatment with fulvestrant in postmenopausal women with advanced breast cancer: experience from a single centre. Endocrine-related cancer. 2006; 13:251-5.

19. deGraffenried LA, Friedrichs WE, Russell DH, Donzis EJ, Middleton AK, Silva JM, Roth RA, Hidalgo M. Inhibition of mTOR activity restores tamoxifen response in breast cancer cells with aberrant Akt Activity. Clinical Cancer Research. 2004; 10:8059-67.

20. Fedele P, Calvani N, Marino A, Orlando L, Schiavone P, Quaranta A, et al. Targeted agents to reverse resistance to endocrine therapy in metastatic breast cancer: where are we now and where are we going? Critical reviews in oncology/ hematology. 2012; 84:243-51.

21. Kumler I, Tuxen MK, Nielsen DL. A systematic review of dual targeting in HER2-positive breast cancer. Cancer treatment reviews. 2014; 40:259-70.

22. Clarke R, Shajahan AN, Riggins RB, Cho Y, Crawford A, Xuan J, Wang Y, Zwart A, Nehra R, Liu MC. Gene network signaling in hormone responsiveness modifies apoptosis and autophagy in breast cancer cells. J Steroid Biochem Mol Biol. 2009; 114:8-20.

23. Chen X, Iliopoulos D, Zhang Q, Tang Q, Greenblatt MB, Hatziapostolou M, Lim E, Tam WL, Ni M, Chen Y, Mai J, Shen H, Hu DZ, et al. XBP1 promotestriple-negative breast cancer by controlling the HIF1 $\alpha$ pathway. Nature. 2014; 508:103-7.

24. Kraskiewicz H, FitzGerald U. InterfERing with endoplasmic reticulum stress. Trends Pharmacol. Sci. 2012; 33:53-63. 